\title{
Flopping-mode electric dipole spin resonance
}

\author{
X. Croot,${ }^{1}$ X. Mi,,${ }^{1,}$ S. Putz,${ }^{1, \dagger}$ M. Benito, ${ }^{2}$ F. Borjans,${ }^{1}$ G. Burkard, ${ }^{2}$ and J. R. Petta $\odot^{1, \sharp}$ \\ ${ }^{1}$ Department of Physics, Princeton University, Princeton, New Jersey 08544, USA \\ ${ }^{2}$ Department of Physics, University of Konstanz, D-78457 Konstanz, Germany
}

(Received 6 May 2019; revised manuscript received 25 November 2019; published 8 January 2020)

\begin{abstract}
Traditional approaches to controlling single spins in quantum dots require the generation of large electromagnetic fields to drive many Rabi oscillations within the spin coherence time. We demonstrate "flopping-mode" electric dipole spin resonance, where an electron is electrically driven in a $\mathrm{Si} / \mathrm{SiGe}$ double quantum dot in the presence of a large magnetic field gradient. At zero detuning, charge delocalization across the double quantum dot enhances coupling to the drive field and enables low-power electric dipole spin resonance. Through dispersive measurements of the single-electron spin state, we demonstrate a nearly three order of magnitude improvement in driving efficiency using flopping-mode resonance, which should facilitate low-power spin control in quantum dot arrays.
\end{abstract}

DOI: 10.1103/PhysRevResearch.2.012006

Recent advances in silicon spin qubits have bolstered their standing as a platform for scalable quantum information processing. As single-qubit gate fidelities exceed $99.9 \%$ [1], twoqubit gate fidelities improve [2-6], and the field accelerates towards large multiqubit arrays [7,8], developing the tools necessary for efficient and scalable spin control is critical [9]. While it is possible to implement single-electron spin resonance in quantum dots (QDs) using ac magnetic fields [10], the requisite high drive powers and associated heat loads are technically challenging and place limitations on attainable Rabi frequencies [11]. As spin systems are scaled beyond a few qubits, methods of spin control which minimize dissipation and reduce qubit crosstalk will be important for low-temperature quantum information processing [12].

Electric dipole spin resonance (EDSR) is an alternative to conventional electron spin resonance. In EDSR, static gradient magnetic fields and oscillating electric fields are used to drive spin rotations [13]. The origin of the effective magnetic field gradient varies across implementations: intrinsic spinorbit coupling [14-16], hyperfine coupling [17], and $g$-factor modulation [18] have been used to couple electric fields to spin states. The inhomogeneous magnetic field generated by a micromagnet $[19,20]$ has been used to create a synthetic spin-orbit field for EDSR, enabling high-fidelity control [1]. Conveniently, this magnetic field gradient gives rise to a spa-

\footnotetext{
*Present address: Google Inc., Santa Barbara, California 93117, USA.

${ }^{\dagger}$ Present address: Vienna Center for Quantum Science and Technology, Universität Wien, 1090 Vienna, Austria.

${ }^{\ddagger}$ petta@princeton.edu
}

Published by the American Physical Society under the terms of the Creative Commons Attribution 4.0 International license. Further distribution of this work must maintain attribution to the author(s) and the published article's title, journal citation, and DOI. tially varying Zeeman splitting, enabling spins in neighboring QDs to be selectively addressed [11,19,21-25].

In this Rapid Communication, we demonstrate a mechanism for driving low-power, coherent spin rotations, which we call "flopping-mode EDSR." In conventional EDSR, the electric drive field couples to a charge trapped in a single quantum dot, leading to a relatively small electronic displacement [16]. We instead drive single spin rotations in a DQD close to zero detuning, $\epsilon=0$, where the electric field can force the electron to flop back and forth between the left and right dots in the "flopping mode," thereby sampling a larger variation in transverse magnetic field.

Neglecting spin, the Hamiltonian describing a singleelectron DQD is $H_{0}=(\epsilon / 2) \tau_{z}+t_{c} \tau_{x}$, where $t_{c}$ is the interdot tunnel coupling and $\tau_{i}$ are the Pauli operators in position $(L, R)$ space [26]. In the highly detuned regime of a DQD (with $|\epsilon| \gg t_{c}$ ), the electron is strongly localized in either the left $|L\rangle$ or right $|R\rangle$ dot with orbital energy $E_{\text {orb }} \approx 3 \mathrm{meV}$ [27]. When $\epsilon=0$ the charge delocalizes across the DQD, leading to the formation of bonding and antibonding states $|\mp\rangle=(|R\rangle \mp|L\rangle) / \sqrt{2}$. Here, the bonding-antibonding energy difference $2 t_{c} \approx 20-40 \mu \mathrm{eV}$ is dominant and the charge is much more susceptible to oscillating electric fields [28,29].

The application of a magnetic field results in Zeeman splitting of the spin states. When the Zeeman energy and $2 t_{c}$ are comparable, the combination of a magnetic field gradient and the large electric dipole moment results in strong spin-charge hybridization. This allows electric fields to couple to spin indirectly via the charge [30-32]. We coherently manipulate a single-electron spin qubit in the flopping-mode regime and find that the power required to drive Rabi oscillations is almost three orders of magnitude less than in single dot EDSR. Additionally, we find a "sweet spot" at $\epsilon=0$ where charge noise is suppressed [33], leading to a fourfold improvement in the qubit quality factor.

The device consists of two single-electron natural$\mathrm{Si} / \mathrm{SiGe}$ DQDs (DQD1 and DQD2) that are embedded in a half-wavelength $\mathrm{Nb}$ superconducting cavity with resonance 
(a)

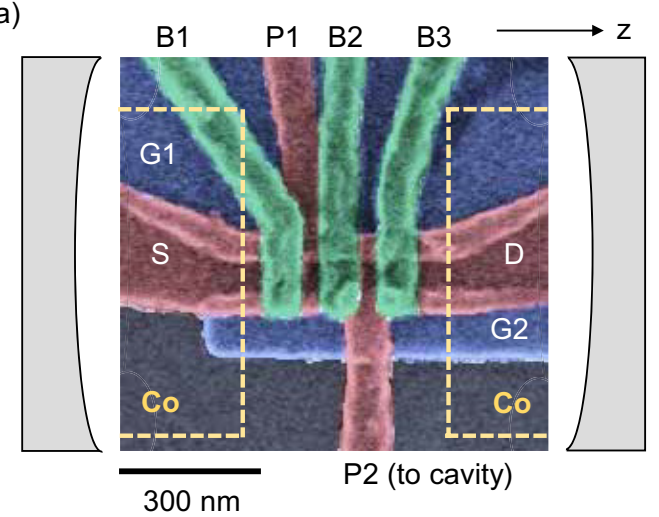

(b)

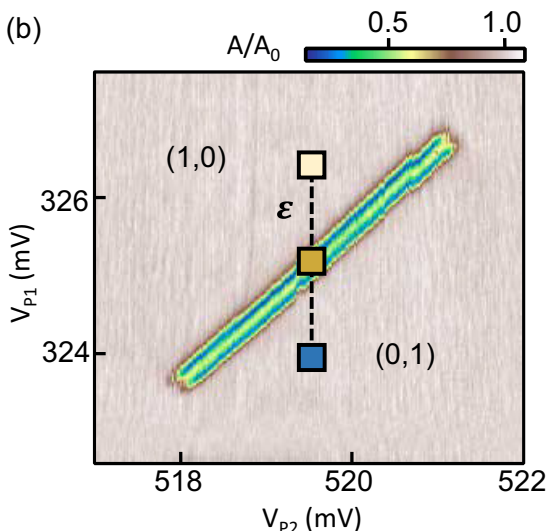

(c)
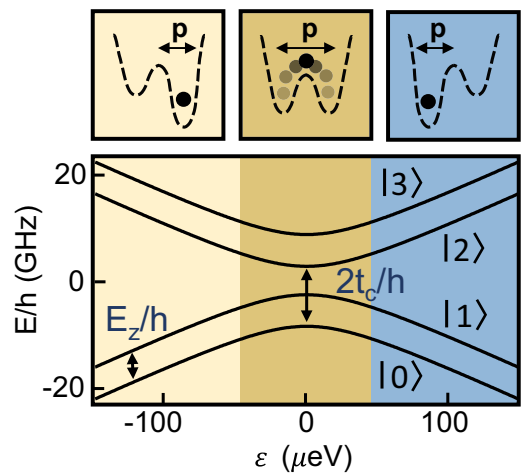

FIG. 1. (a) False-color SEM image of the device. The locations of the cobalt micromagnets are indicated by the yellow dashed lines. Gate $\mathrm{P} 2$ is galvanically connected to the center pin of the superconducting cavity. (b) Normalized cavity transmission amplitude $A / A_{0}$ as a function of the gate voltages $V_{\mathrm{P} 1}$ and $V_{\mathrm{P} 2}$ near the interdot charge transition for DQD2, with $2 t_{c} / h=4.9 \mathrm{GHz}$. The dashed line denotes the DQD detuning axis. (c) Top: Schematic comparison of the far detuned single dot regime $\left(|\epsilon| \gg 2 t_{c}\right.$ ) and flopping-mode regime $(\epsilon \approx 0)$. Charge hybridization near $\epsilon=0$ results in a comparatively large electric dipole $p$. Bottom: Energy diagram calculated with $2 t_{c} / h=11.1 \mathrm{GHz}, B_{z}=209.4 \mathrm{mT}$, $b_{z}=0 \mathrm{mT}$, and $b_{x}=15 \mathrm{mT}$.

frequency $f_{c}=5.846 \mathrm{GHz}$ [31]. A false-color scanning electron microscope (SEM) image of one of the DQDs is shown in Fig. 1(a). Electronic confinement is achieved using an overlapping aluminum gate stack [27]. For each DQD, the plunger gate $\mathrm{P} 2$ is connected to the center pin of the superconducting cavity, efficiently coupling the charge confined in the DQD to the electric field of the cavity. Only one DQD is active at a time.

Cobalt micromagnets [yellow dashed lines in Fig. 1(a)] generate a local magnetic field gradient with longitudinal and transverse components. The total magnetic field at the device $\vec{B}^{\text {tot }}$ is the sum of the external magnetic field $\vec{B}^{\text {ext }}$ applied in the $z$ direction, and the stray field from the micromagnet $\vec{B}^{\mathrm{M}}$. As a result, the total magnetic field $\vec{B}^{\text {tot }}$ is a sensitive function of position in the DQD confinement potential. The micromagnet enables EDSR $[19,20]$ and gives rise to spin-photon coupling, which is used here for dispersive readout of the spin state [31,34].

We first probe the charge degree of freedom with $B^{\text {tot }}=0$ by measuring the cavity transmission amplitude $A / A_{0}$ in the single-photon regime, as a function of the gate voltages $V_{\mathrm{P} 1}$ and $V_{\mathrm{P} 2}$ [see Fig. 1(b)]. These data are acquired near the $(1,0)-(0,1)$ interdot charge transition with the probe frequency equal to the cavity frequency $f=f_{c}$. Here, $\left(n_{L}, n_{R}\right)$ denotes the charge occupancy of the DQD, where $n_{L}\left(n_{R}\right)$ is the number of electrons in the left (right) dot. Around $\epsilon=0$, where the DQD is maximally polarizable, electric fields from the cavity result in charge dynamics within the DQD that load the superconducting cavity and reduce $A / A_{0}[34,35]$. Neglecting spin, the energy difference between the bonding and antibonding charge states is $\Omega(\epsilon)=\sqrt{\epsilon^{2}+4 t_{c}^{2}}$. In the case where $2 t_{c}<h f_{c}$, there will be two values of detuning where $\Omega(\epsilon)=h f_{c}$ ( $h$ is Planck's constant). Around these values of detuning the cavity response is substantial, as is evident from the data in Fig. 1(b) [34,35]. The flopping-mode EDSR mechanism is most effective near $\epsilon=0$, where the electric dipole moment $p$ is largest [Fig. 1(c)].
In the presence of a micromagnet and external magnetic field, the Hamiltonian describing the single-electron DQD is

$$
H_{s}=H_{0}+\frac{1}{2} g \mu_{B}\left[B_{z} \sigma_{z}+\left(b_{x} \sigma_{x}+b_{z} \sigma_{z}\right) \tau_{z}\right],
$$

where $\sigma_{i}$ are the Pauli operators in spin space, $B_{z}$ is the homogeneous magnetic field component in the $z$ direction, $g=2$ is the electronic $g$-factor, and $\mu_{B}$ is the Bohr magneton [36]. In general, $\vec{B}^{\mathrm{M}}$ will generate longitudinal $b_{z}$ and transverse $\left(b_{x}, b_{y}\right)$ gradients that will modify the energy level spectrum. We define $2 b_{i}(i=x, y, z)$ as the difference in total magnetic field between the left and right dots in the $i$ direction. Without loss of generality, we take $b_{y}=0$ in the remainder of this Rapid Communication [37]. The total magnetic field components at the left and right dots can be written as $B_{z}^{\text {tot }}=B_{z} \pm b_{z}$ and $B_{x}^{\text {tot }}=B_{x} \pm b_{x}$, where $B_{z(x)}$ is the homogeneous magnetic field in the $z(x)$ direction. For our micromagnet design, we expect $B_{x} \approx 0$, but note that in the case where $B_{x} \neq 0$, the geometric coordinate system can always be rotated to satisfy $B_{x}=0$ [37]. The Zeeman energy is given by $E_{z}=g \mu_{B} B_{z}$.

Zeeman splitting of the bonding/antibonding states leads to the four-level system shown in Fig. 1(c), where $|0(1)\rangle \equiv$ $|-, \downarrow(\uparrow)\rangle$ refer to the spin-down and spin-up bonding states, and $|2(3)\rangle \equiv|+, \downarrow(\uparrow)\rangle$ to the spin-down and spin-up antibonding states. Spin-preserving interdot tunnel coupling $t_{c}$ results in the anticrossings near $\epsilon=0$. The $b_{z}$ component gives rise to a spatially varying longitudinal field such that the energy splitting $E_{01}$ between the ground $|0\rangle$ and first excited state $|1\rangle$ can vary significantly in the far detuned limits, e.g., $|\epsilon| \gg 2 t_{c}$. The transverse components of the micromagnet field hybridize the spin-orbital states near $\epsilon=0$ and lead to spin-photon coupling [31,36], which enables dispersive spin state readout [34].

In general, the electron spin resonance condition will be a function of detuning owing to the magnetic field gradients. To investigate the flopping-mode EDSR mechanism, we first map out the spin resonance condition by measuring the 

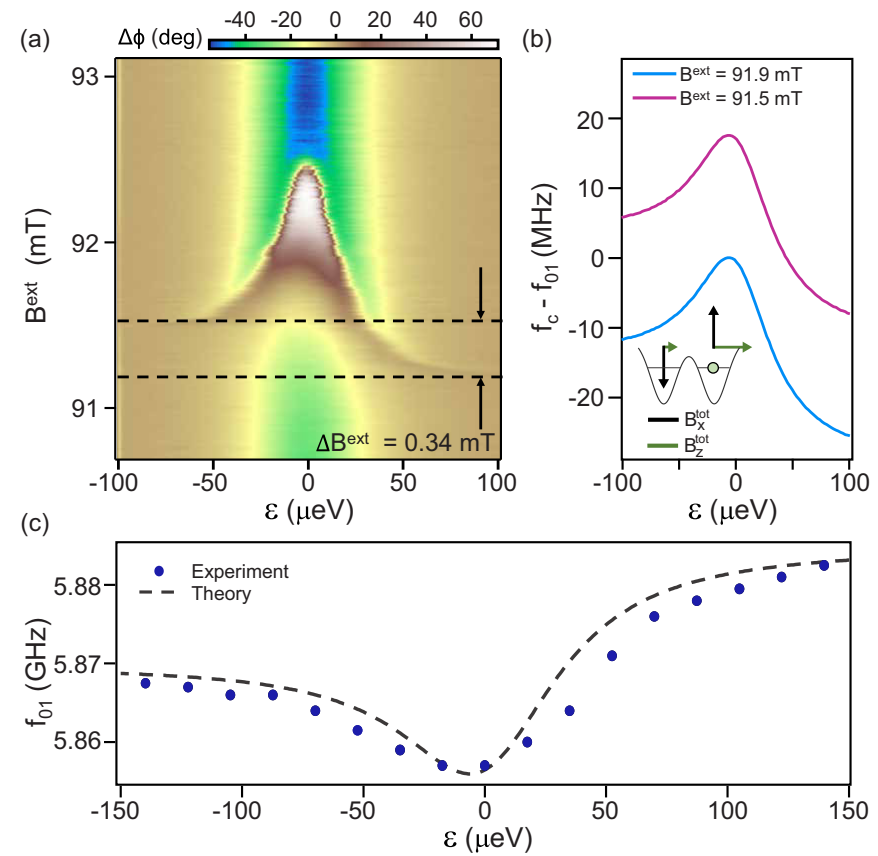

FIG. 2. (a) Phase response of the cavity transmission $\Delta \phi$ as a function of $B^{\text {ext }}$ and $\epsilon$ for DQD1 with $2 t_{c} / h=7.4 \mathrm{GHz}$. Dashed lines show asymmetry in spin-cavity interactions at high and low detuning. The extracted $\Delta B^{\text {ext }}$ is used to determine $b_{z}$. (b) Difference between the cavity frequency $f_{c}$ and spin transition frequency $f_{01}$ as a function of $\epsilon$ for $B^{\text {ext }}=91.9 \mathrm{mT}$ (blue) and $B^{\text {ext }}=91.5 \mathrm{mT}$ (purple). Inset: Cartoon of a DQD in the presence of spatially varying $B_{x}^{\text {tot }}\left(B_{z}^{\text {tot }}\right)$ fields, not drawn to scale. (c) $f_{01}$ as a function of $\epsilon$ for DQD1, with $2 t_{c} / h=11.1 \mathrm{GHz}$, as extracted from time-domain Rabi oscillations. The dashed line shows a fit to theory with $2 t_{c} / h=11.1 \mathrm{GHz}, B_{z}=$ $209.4 \mathrm{mT}, b_{x}=15 \mathrm{mT}$, and $b_{z}=0.27 \mathrm{mT}$.

cavity phase shift $\Delta \phi$ as a function of $B^{\text {ext }}$ and $\epsilon$ [Fig. 2(a)]. The funnel-shaped feature in Fig. 2(a) is a consequence of detuning-dependent charge hybridization and Zeeman physics $[32,36]$ in the regime where $E_{01} \ll 2 t_{c}$. At low $B^{\text {ext }}$, the spin transition is detuned from the cavity, but there is still a small phase response around $\epsilon=0$ due to the large electric dipole moment $[34,35]$. At large detunings $\left(|\epsilon| \gg 2 t_{c}\right)$ the energy splitting $E_{01}$ is dominated by Zeeman physics. At small detunings, levels $|1\rangle$ and $|2\rangle$ hybridize due to transverse magnetic fields [36], which pulls $E_{01}$ slightly below the Zeeman energy. As a result, when $E_{01}$ is slightly less than $h f_{c}$ at $\epsilon=0$, there are two values of finite detuning for which $E_{01}$ is on resonance with the cavity, giving rise to the wings of the funnel-shaped feature that begins at $B^{\text {ext }} \sim 91.2 \mathrm{mT}$. As $B^{\text {ext }}$ increases further the values of detuning that lead to resonance with the cavity shift closer to $\epsilon=0$. Eventually, at $B^{\text {ext }} \sim 91.9 \mathrm{mT}$, the two resonance conditions merge at $\epsilon=0$. Figure 2(b) shows theoretical predictions for $f_{c}-f_{01}$ as a function of $\epsilon$ for $B^{\text {ext }}=91.5$ and $91.9 \mathrm{mT}$, with $f_{01} \equiv E_{01} / h$.

With the electron spin resonance frequency $f_{01}$ now mapped out as a function of $B^{\text {ext }}$ and $\epsilon$, we can drive coherent single spin rotations using flopping-mode EDSR. At $\epsilon=0$, a microwave burst of frequency $f_{s}$ and duration $\tau_{B}$ is applied to gate $\mathrm{P} 1$ to drive coherent spin rotations. The final spin state is

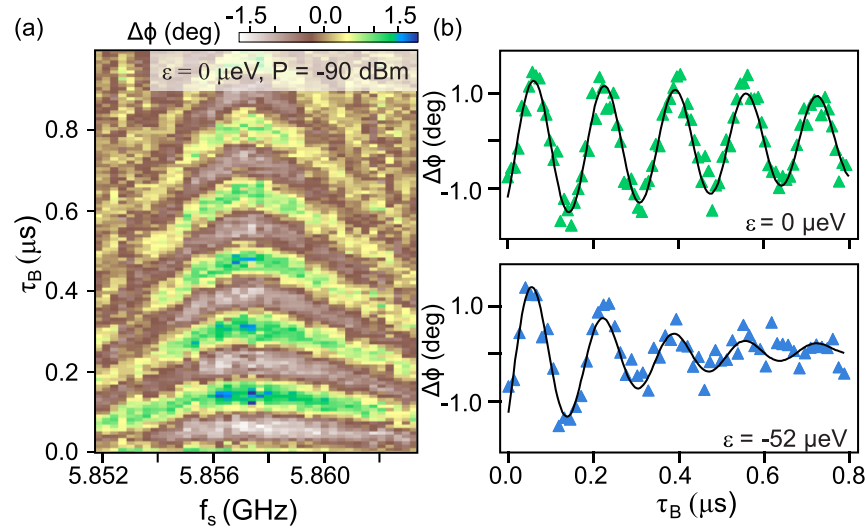

FIG. 3. (a) Rabi chevron acquired at $\epsilon=0,2 t_{c} / h=11.1 \mathrm{GHz}$, and with gate $\mathrm{P} 1$ driven at frequency $f_{s}$ for a time $\tau_{B}$. The cavity phase response $\Delta \phi$ is measured for $20 \mu$ s after each Rabi burst. Data are acquired with a total per point integration time of $100 \mathrm{~ms}$. (b) Rabi oscillations acquired with $\epsilon=0$ (upper panel, $P_{s}=-90 \mathrm{dBm}$ ) and $\epsilon=-52 \mu \mathrm{eV}$ (lower panel, $P_{s}=-83 \mathrm{dBm}$ ). The data are fit to an exponentially decaying sinusoid (solid black line). Data are from DQD1.

read out dispersively at $\epsilon=0$ by measuring the cavity phase response $\Delta \phi[31]$.

The spin transition frequency $f_{01}$ is extracted from the center frequency of Rabi chevron data, and plotted in Fig. 2(c) as a function of $\epsilon$. When $2 t_{c} \gg E_{z}$, the lowest $f_{01}$ occurs near $\epsilon=0$ due to spin-charge hybridization, and $f_{01}$ increases as $|\epsilon|$ increases. The trends in these data are in general agreement with the data measured using microwave spectroscopy in Fig. 2(a). The asymmetry of the data in Figs. 2(a) and 2(c) about $\epsilon=0$ is due to the longitudinal gradient field $b_{z}$. The difference in external field $\Delta B^{\text {ext }}$ [given by the splitting of the dashed lines in Fig. 2(a)] required to bring the spin onto resonance with the cavity at large negative and positive detunings provides a measure of $b_{z}$. From the data, we find that $\Delta B^{\text {ext }}=0.34 \mathrm{mT}$, and using the expression $2 b_{z}=(1+$ $\chi) \Delta B^{\text {ext }}$, where $\chi$ is the micromagnet magnetic susceptibility [31], we extract $b_{z}=0.27 \mathrm{mT}$. We take the extracted value of $b_{z}$ and fit the data in Fig. 2(c), finding good agreement between experiment and our theoretical model.

Having gained a quantitative understanding of how $f_{01}$ depends on $\epsilon$, we now compare EDSR in the flopping-mode and single dot regimes. A typical flopping-mode data set is shown in Fig. 3(a), where $\Delta \phi$ is plotted as a function of $f_{s}$ and $\tau_{B}$. As expected, the Rabi oscillation visibility is maximal when $f_{s}$ is resonant with $f_{01}$. By simultaneously applying a microwave burst and square pulse to gate P1, we can drive coherent spin rotations at a value of detuning set by the amplitude of the square pulse. Due to the ratio of electric dipole moments in these regimes, the power $P$ required to drive fast coherent rotations in the single dot regime is expected to be much higher. As shown in the upper panel of Fig. 3(b), at $\epsilon=0$ a Rabi frequency $f_{\text {Rabi }} \approx 6 \mathrm{MHz}$ is achieved with $P=-90 \mathrm{dBm}$ at the device. In contrast, when $\epsilon=-52 \mu \mathrm{eV}$ a power of $P=-83 \mathrm{dBm}$ is required to achieve approximately the same Rabi frequency [see Fig. 3(b), lower panel]. Power adjustments are implemented manually. 

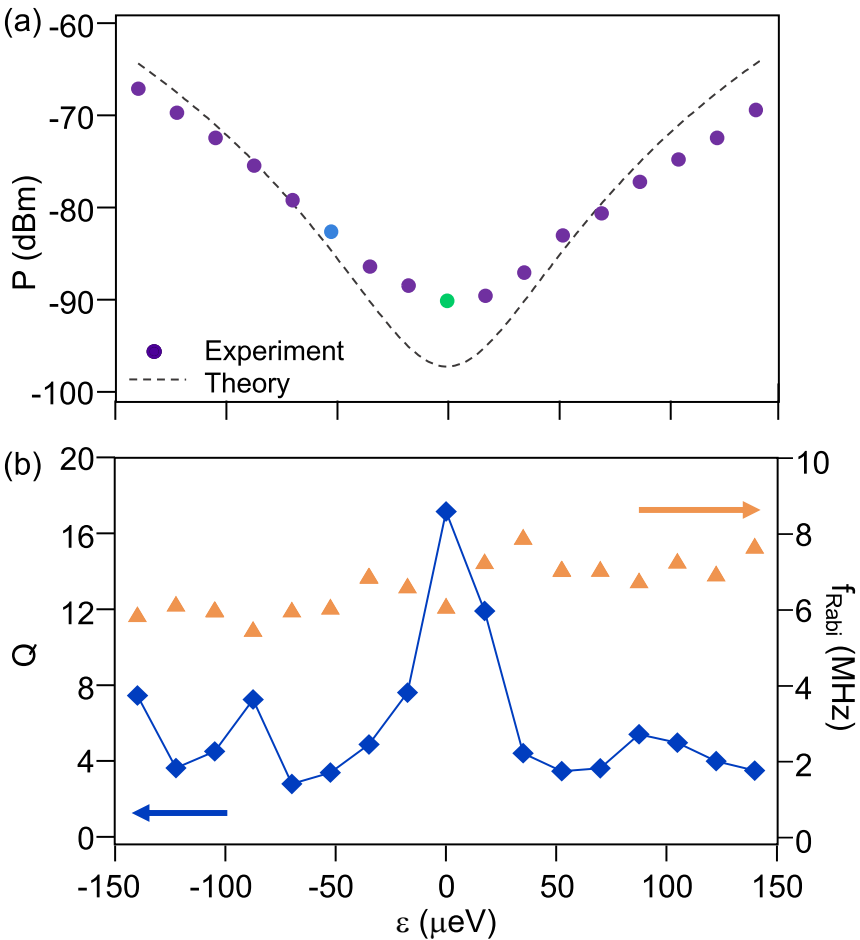

FIG. 4. (a) On-chip power $P$ required to drive $\simeq 6 \mathrm{MHz}$ Rabi oscillations as a function of $\epsilon$, with $2 t_{c} / h=11.1 \mathrm{GHz}$. Green (light blue) data points at $\epsilon=0(\epsilon=-52 \mu \mathrm{eV})$ are taken from the same data set as the top (bottom) panel in Fig. 3(b). The dashed line is a theoretical fit. (b) Quality factor $Q$ of Rabi oscillations and Rabi frequency $f_{\text {Rabi }}$ plotted as a function of $\epsilon$. There is a significant improvement in $Q$ around $\epsilon=0$.

The actual power at the gate is determined by measuring the envelope of Landau-Zener-Stueckelberg interference fringes as a function of increasing $P$ [38]. The $V_{\text {rms }}$ measured at the gate is then converted to power via $V_{\text {rms }}=2 \sqrt{P Z_{0}}$, where $Z_{0}=50 \Omega$ and the factor of 2 accounts for the high impedance termination of the gate line. We fit the Rabi oscillations to an exponentially decaying sinusoid with $T_{2}^{\text {Rabi }}=1.4 \mu \mathrm{s}$ at $\epsilon=0 \mu \mathrm{eV}$ and $T_{2}^{\mathrm{Rabi}}=0.24 \mu \mathrm{s}$ at $\epsilon=-52 \mu \mathrm{eV}$.

The full crossover from the single dot to the floppingmode regime is examined over a $300 \mu \mathrm{eV}$ range of detuning in Fig. 4(a). Here, we plot the power required to achieve $\simeq 6 \mathrm{MHz}$ Rabi oscillations as a function of $\epsilon$. The data are nearly symmetric about $\epsilon=0$, as expected from the detuning symmetry of the energy levels. Most importantly, these data show that $\sim 250 \times$ less microwave power is required to achieve a $f_{\text {Rabi }} \approx 6-8 \mathrm{MHz}$ at $\epsilon=0$ compared to the standard single dot EDSR regime. We fit the data in Fig. 4(a) to theory developed in Ref. [37], leaving the lever arm $\alpha$ as a free parameter, and fixing the estimated interdot spacing $d=$
$100 \mathrm{~nm}$, the orbital energy $E_{\text {orb }}=3 \mathrm{meV}$ [27], $b_{x}=15 \mathrm{mT}$, $B_{z}=209.4 \mathrm{mT}, 2 t_{c} / h=11.1 \mathrm{GHz}$, and $f_{\text {Rabi }}=6 \mathrm{MHz}$. We use an effective mass $m_{e}^{*}=0.19 m_{e}$, where $m_{e}$ is the freeelectron mass. The ac voltage at the gate $V_{\mathrm{P} 1}^{\mathrm{ac}}$ is converted to electric field $E_{\mathrm{ac}}$ at the DQD via $E_{\mathrm{ac}}=\alpha V_{\mathrm{P} 1}^{\mathrm{ac}} / e d$. We find a best fit lever arm of $\alpha=0.06 \mathrm{eV} / V_{\mathrm{P} 1}$, and obtain good agreement between experiment and theory.

A charge noise sweet spot exists when $\partial E_{01} / \partial \epsilon=0$ [33]. In the absence of a longitudinal $\left(b_{z}\right)$ gradient the sweet spot occurs at $\epsilon=0$. Theory predicts that a $b_{z}$ gradient may shift the sweet spot to finite detuning, or if the gradient field is large enough, destroy it entirely [37]. We search for evidence of a sweet spot by examining the quality factor of $\pi$ rotations $Q \equiv 2 T_{2}^{\mathrm{Rabi}} f_{\text {Rabi }}$ as a function of detuning [1]. At each value of detuning, a Rabi chevron is acquired with $P$ set to achieve $f_{\text {Rabi }} \approx 6 \mathrm{MHz}$, similar to Fig. 3(a). We take a Fourier transform of each column of the chevron and identify $f_{01}$. At this $f_{01}$, we fit the Rabi oscillations as a function of $\tau_{B}$ to extract $T_{2}^{\text {Rabi }}$ and $f_{\text {Rabi }}$.

The Rabi frequency and $Q$ factor are plotted as a function of $\epsilon$ in Fig. 4(b). At finite detuning $Q \approx 4$. We observe more than a fourfold increase in the quality factor, with $Q=18$ at $\epsilon=0$. The enhancement of $Q$ in the flopping-mode regime can be attributed to the presence of the charge noise sweet spot, which to first order decouples the spin from electrical detuning noise. While the $Q$ factors achieved here are lower than those reported elsewhere [1,5], from theoretical models [37] we expect that for an optimized $t_{c}$, fabricating devices on enriched ${ }^{28} \mathrm{Si}$ quantum wells and reducing charge noise will yield quality factors comparable to those cited for conventional EDSR [1]. Similar to other work [1], we observe a deterioration in $Q$ at high drive powers.

In summary, we demonstrate an efficient flopping-mode mechanism for EDSR in semiconductor DQDs. Compared to single dot EDSR, flopping-mode EDSR requires nearly three orders of magnitude less power, rendering it a valuable control technique for future spin-based quantum processors. Conveniently, the flopping-mode regime of maximal power efficiency coincides with a charge noise sweet spot, yielding a fourfold improvement in qubit quality factor. While the device studied here is embedded in a microwave cavity, flopping-mode EDSR could be implemented in DQDs that are read out using conventional spin-to-charge conversion [39] or Pauli blockade [40]. We anticipate that flopping-mode spin resonance will enable power-efficient single-qubit control in large-scale silicon quantum processors.

This research was sponsored by ARO Grant No. W911NF15-1-0149 and the Gordon and Betty Moore Foundation's EPiQS Initiative through Grant No. GBMF4535. Devices were fabricated in the Princeton University Quantum Device Nanofabrication Laboratory.
[1] J. Yoneda, K. Takeda, T. Otsuka, T. Nakajima, Ma. R. Delbecq, G. Allison, T. Honda, T. Kodera, S. Oda, Y. Hoshi, N. Usami, K. M. Itoh, and S. Tarucha, A quantum-dot spin qubit with coherence limited by charge noise and fidelity higher than 99.9\%, Nat. Nanotechnol. 13, 102 (2018).
[2] M. Veldhorst, C. H. Yang, J. C. C. Hwang, W. Huang, J. P. Dehollain, J. T. Muhonen, S. Simmons, A. Laucht, F. E. Hudson, K. M. Itoh, A. Morello, and A. S. Dzurak, A two-qubit logic gate in silicon, Nature (London) 526, 410 (2015). 
[3] X. Xue, T. F. Watson, D. R. Ward, D. E. Savage, M. G. Lagally, S. N. Coppersmith, M. A. Eriksson, S. Wehner, and L. M. K. Vandersypen, Benchmarking Gate Fidelities in a Si/SiGe TwoQubit Device, Phys. Rev. X 9, 021011 (2019).

[4] W. Huang, C. H. Yang, K. W. Chan, T. Tanttu, B. Hensen, R. C. C. Leon, M. A. Fogarty, J. C. C. Hwang, F. E. Hudson, K. M. Itoh, A. Morello, A. Laucht, and A. S. Dzurak, Fidelity benchmarks for two-qubit gates in silicon, Nature (London) 569, 532 (2019).

[5] D. M. Zajac, A. J. Sigillito, M. Russ, F. Borjans, J. M. Taylor, G. Burkard, and J. R. Petta, Resonantly driven CNOT gate for electron spins, Science 359, 439 (2018).

[6] T. F. Watson, S. G. J. Philips, E. Kawakami, D. R. Ward, P. Scarlino, M. Veldhorst, D. E. Savage, M. G. Lagally, M. Friesen, S. N. Coppersmith, M. A. Eriksson, and L. M. K. Vandersypen, A programmable two-qubit quantum processor in silicon, Nature (London) 555, 633 (2018).

[7] P.-A. Mortemousque, E. Chanrion, B. Jadot, H. Flentje, A. Ludwig, A. D. Wieck, M. Urdampilleta, C. Bauerle, and T. Meunier, Coherent control of individual electron spins in a two dimensional array of quantum dots, arXiv:1808.06180.

[8] A. R. Mills, D. M. Zajac, M. J. Gullans, F. J. Schupp, T. M. Hazard, and J. R. Petta, Shuttling a single charge across a onedimensional array of silicon quantum dots, Nat. Commun. 10, 1063 (2019).

[9] R. Hanson, L. P. Kouwenhoven, J. R. Petta, S. Tarucha, and L. M. K. Vandersypen, Spins in few-electron quantum dots, Rev. Mod. Phys. 79, 1217 (2007).

[10] F. H. L. Koppens, C. Buizert, K. J. Tielrooij, I. T. Vink, K. C. Nowack, T. Meunier, L. P. Kouwenhoven, and L. M. K. Vandersypen, Driven coherent oscillations of a single electron spin in a quantum dot, Nature (London) 442, 766 (2006).

[11] K. Takeda, J. Kamioka, T. Otsuka, J. Yoneda, T. Nakajima, M. R. Delbecq, S. Amaha, G. Allison, T. Kodera, S. Oda, and $\mathrm{S}$. Tarucha, A fault-tolerant addressable spin qubit in a natural silicon quantum dot, Sci. Adv. 2, e1600694 (2016).

[12] J. M. Hornibrook, J. I. Colless, I. D. Conway Lamb, S. J. Pauka, H. Lu, A. C. Gossard, J. D. Watson, G. C. Gardner, S. Fallahi, M. J. Manfra, and D. J. Reilly, Cryogenic Control Architecture for Large-Scale Quantum Computing, Phys. Rev. Applied 3, 024010 (2015).

[13] E. I. Rashba and A. L. Efros, Orbital Mechanisms of ElectronSpin Manipulation by an Electric Field, Phys. Rev. Lett. 91, 126405 (2003).

[14] V. N. Golovach, M. Borhani, and D. Loss, Electric-dipoleinduced spin resonance in quantum dots, Phys. Rev. B. 74, 165319 (2006).

[15] C. Flindt, A. S. Sorensen, and K. Flensberg, Spin-Orbit Mediated Control of Spin Qubits, Phys. Rev. Lett. 97, 240501 (2006).

[16] K. C. Nowack, F. H. L. Koppens, Yu. V. Nazarov, and L. M. K. Vandersypen, Coherent control of a single electron spin with electric fields, Science 318, 1430 (2007).

[17] E. A. Laird, C. Barthel, E. I. Rashba, C. M. Marcus, M. P. Hanson, and A. C. Gossard, Hyperfine-Mediated Gate-Driven Electron Spin Resonance, Phys. Rev. Lett. 99, 246601 (2007).

[18] Y. Kato, R. C. Myers, D. C. Driscoll, A. C. Gossard, J. Levy, and D. D. Awschalom, Gigahertz electron spin manipulation using voltage-controlled $g$-tensor modulation, Science 299, 1201 (2003).
[19] M. Pioro-Ladrière, T. Obata, Y. Tokura, Y. S. Shin, T. Kubo, K. Yoshida, T. Taniyama, and S. Tarucha, Electrically driven single-electron spin resonance in a slanting Zeeman field, Nat. Phys. 4, 776 (2008).

[20] E. Kawakami, P. Scarlino, D. R. Ward, F. R. Braakman, D. E Savage, M. G. Lagally, Mark Friesen, S. N. Coppersmith, M. A. Eriksson, and L. M. K. Vandersypen, Electrical control of a long-lived spin qubit in a $\mathrm{Si} / \mathrm{SiGe}$ quantum dot, Nat. Nanotechnol. 9, 666 (2014).

[21] T. Obata, M. Pioro-Ladrière, Y. Tokura, Y. S. Shin, T. Kubo, K. Yoshida, T. Taniyama, and S. Tarucha, Coherent manipulation of individual electron spin in a double quantum dot integrated with a micromagnet, Phys. Rev. B 81, 085317 (2010).

[22] S. Nadj-Perge, S. M. Frolov, E. P. A. M. Bakkers, and L. P. Kouwenhoven, Spin-orbit qubit in a semiconductor nanowire, Nature (London) 468, 1084 (2010).

[23] J. Yoneda, T. Otsuka, T. Nakajima, T. Takakura, T. Obata, M. Pioro-Ladriere, H. Lu, C. J. Palmstrom, A. C. Gossard, and S. Tarucha, Fast Electrical Control of Single Electron Spins in Quantum Dots with Vanishing Influence from Nuclear Spins, Phys. Rev. Lett. 113, 267601 (2014).

[24] A. Noiri, J. Yoneda, T. Nakajima, T. Otsuka, M. R. Delbecq, K. Takeda, S. Amaha, G. Allison, A. Ludwig, A. D. Wieck, and S. Tarucha, Coherent electron-spin-resonance manipulation of three individual spins in a triple quantum dot, Appl. Phys. Lett. 108, 153101 (2016).

[25] T. Ito, T. Otsuka, T. Nakajima, M. R. Delbecq, S. Amaha, J. Yoneda, K. Takeda, A. Noiri, G. Allison, A. Ludwig, A. D. Wieck, and S. Tarucha, Four single-spin Rabi oscillations in a quadruple quantum dot, Appl. Phys. Lett. 113, 093102 (2018).

[26] T. Hayashi, T. Fujisawa, H. D. Cheong, Y. H. Jeong, and Y. Hirayama, Coherent Manipulation of Electronic States in a Double Quantum Dot, Phys. Rev. Lett. 91, 226804 (2003).

[27] D. M. Zajac, T. M. Hazard, X. Mi, E. Nielsen, and J. R. Petta, Scalable Gate Architecture for a One-Dimensional Array of Semiconductor Spin Qubits, Phys. Rev. Applied 6, 054013 (2016).

[28] X. D. Hu, Y. X. Liu, and F. Nori, Strong coupling of a spin qubit to a superconducting stripline cavity, Phys. Rev. B 86, 035314 (2012).

[29] D. Kim, D. R. Ward, C. B. Simmons, J. K. Gamble, R. BlumeKohout, E. Nielsen, D. E. Savage, M. G. Lagally, M. Friesen, S. N. Coppersmith, and M. A. Eriksson, Microwave-driven coherent operation of a semiconductor quantum dot charge qubit, Nat. Nanotechnol. 10, 243 (2015).

[30] J. J. Viennot, M. C. Dartiailh, A. Cottet, and T. Kontos, Coherent coupling of a single spin to microwave cavity photons, Science 349, 408 (2015).

[31] X. Mi, M. Benito, S. Putz, D. M. Zajac, J. M. Taylor, G. Burkard, and J. R. Petta, A coherent spin-photon interface in silicon, Nature (London) 555, 599 (2018).

[32] N. Samkharadze, G. Zheng, N. Kalhor, D. Brousse, A. Sammak, U. C. Mendes, A. Blais, G. Scappucci, and L. M. K. Vandersypen, Strong spin-photon coupling in silicon, Science 359, 1123 (2018).

[33] D. Vion, A. Aassime, A. Cottet, P. Joyez, H. Pothier, C. Urbina, D. Esteve, and M. H. Devoret, Manipulating the quantum state of an electrical circuit, Science 296, 886 (2002).

[34] K. D. Petersson, L. W. McFaul, M. D. Schroer, M. Jung, J. M. Taylor, A. A. Houck, and J. R. Petta, Circuit quantum 
electrodynamics with a spin qubit, Nature (London) 490, 380 (2012).

[35] T. Frey, P. J. Leek, M. Beck, A. Blais, T. Ihn, K. Ensslin, and A. Wallraff, Dipole Coupling of a Double Quantum Dot to a Microwave Resonator, Phys. Rev. Lett. 108, 046807 (2012).

[36] M. Benito, X. Mi, J. M. Taylor, J. R. Petta, and G. Burkard, Input-output theory for spin-photon coupling in Si double quantum dots, Phys. Rev. B 96, 235434 (2017).

[37] M. Benito, X. Croot, C. Adelsberger, S. Putz, X. Mi, J. R. Petta, and G. Burkard, Electric-field control and noise protection of the flopping-mode spin qubit, Phys. Rev. B 100, 125430 (2019).
[38] S. N. Shevchenko, S. Ashhab, and F. Nori, LandauZener-Stuckelberg interferometry, Phys. Rep. 492, 1 (2010).

[39] J. M. Elzerman, R. Hanson, L. H. Willems van Beveren, B. Witkamp, L. M. K. Vandersypen, and L. P. Kouwenhoven, Single-shot read-out of an individual electron spin in a quantum dot, Nature (London) 430, 431 (2004).

[40] J. R. Petta, A. C. Johnson, J. M. Taylor, E. A. Laird, A. Yacoby, M. D. Lukin, C. M. Marcus, M. P. Hanson, and A. C. Gossard, Coherent manipulation of coupled electron spins in semiconductor quantum dots, Science 309, 2180 (2005). 